\title{
Rectal Necrosis, CTCAE
}

National Cancer Institute

\section{Source}

National Cancer Institute. Rectal Necrosis, CT CAE. NCI Thesaurus. Code C57863.

A disorder characterized by a necrotic process occurring in the rectal wall. 